\title{
Artificial Neural Networks Analysis of
} polysomnographic and clinical features in Pediatric
Acute-Onset Neuropsychiatric Syndrome (PANS):
from sleep alteration to "Brain Fog"

\author{
Antonella Gaglianol,* \\ Monica Puligheddu (iD) ${ }^{2, *}$ \\ Nadia Ronzano ${ }^{3}$ \\ Patrizia Congiu (iD ${ }^{2}$ \\ Marcello Giuseppe Tanca ${ }^{3}$ \\ Ida Cursio (I) $^{3}$ \\ Sara Carucci ${ }^{1}$ \\ Stefano Sotgiu ${ }^{4}$ \\ Enzo Grossi ${ }^{5}$ \\ Alessandro Zuddas ${ }^{1,3}$ \\ 'Child \& Adolescent Neuropsychiatry \\ Unit, "Azienda Ospedaliera Brotzu" \\ Hospital Trust, Cagliari, Italy; ${ }^{2}$ Sleep \\ Disorder Centre, Department of Medical \\ Sciences and Public Health, University of \\ Cagliari, Cagliari, Italy; ${ }^{3}$ Section of \\ Neuroscience \& Clinical Pharmacology, \\ Department of Biomedical Sciences, \\ University of Cagliari, Cagliari, Italy; \\ ${ }^{4}$ Child Neuropsychiatry Unit, \\ Department of Medical, Surgical and \\ Experimental Sciences, University \\ Hospital of Sassari, Sassari, Italy; ${ }^{5}$ Autism \\ Research Unit, Villa Santa Maria \\ Foundation, Como, Italy \\ *These authors contributed equally to \\ this work
}

Study Objectives: PANS (pediatric acute onset neuropsychiatric syndrome) is thought to be the result of several mechanisms and multiple etiologies, ranging from endocrine/metabolic causes to postinfectious autoimmune and neuroinflammatory disorders. Sleep disorders represent one of the most frequent manifestations of PANS, involving around $80 \%$ of patients. The present study describes the clinical and polysomnographic features in a group of PANS children identifying the relationships between sleep disorders and other PANS symptoms.

Methods: All participants underwent a clinical evaluation including comprehensive sleep history, polysomnography, cognitive assessment and blood chemistry examination. A data mining approach with fourth-generation artificial neural networks has been used in order to discover subtle trends and associations among variables.

Results: Polysomnography showed abnormality in 17 out of 23 recruited subjects $(73.9 \%)$. In particular, 8/17 children (47\%) had ineffective sleep, 10/17 (58.8\%) fragmented sleep, 8/ 17 (47.1\%) periodic limb movement disorder (PLMD) and 11/17 (64.7\%) REM-sleep without atonia (RSWA). Most subjects presented more than one sleep disturbances. Notably, among the 19/23 patients diagnosed with Tic/Tourette disorder, 8/19 (42.1\%) show PLMD and 10/19 (52.6\%) RSWA. Artificial neural network methodology and the auto-contractive map exploited the links among the full spectrum of variables revealing the simultaneous connections among them, facing the complexity of PANS phenotype.

Conclusion: Disordered sleep represents, for prevalence and impact on quality of life, a cardinal symptom in patients with PANS. Thus, considering the weight of sleep disturbances on diagnosis and prognosis of PANS, we could consider the possibility of including them among the major diagnostic criteria.

Keywords: PANS, pediatric acute onset neuropsychiatric syndrome, sleep disorders, Tourette disorder, polysomnography, auto-contractive map

\section{Plain Language Summary}

a) Current Knowledge/Study Rationale

Sleep Disorders are one of the most frequent manifestations in PANS, involving up to $80 \%$ of patients, but to date few studies have been conducted analyzing the sleep characteristics of these patients.

The aim of this study was to describe the clinical and polysomnographic characteristics of sleep and identify any sleep disorders in a population of children affected by PANS. 


\section{b) Study Impact}

Sleep alterations represent, for prevalence and impact on quality of life, a cardinal symptom of PANS phenotype.

Sleep should always be evaluated in PANS patients, and when disordered sleep on daytime sleepiness are reported, a PSG recording should be performed.

\section{Introduction}

PANS (Pediatric Acute Onset Neuropsychiatric Syndrome) is a recently described syndrome characterized by an abrupt and acute onset of obsessive-compulsive symptoms and/or food restriction in association with at least 2 of the following symptoms: anxiety, emotional lability, depression, irritability, aggression, oppositionality, deterioration in school performance, behavioural regression, sensory and movement abnormalities, sleep disturbances, and increased urinary frequency. ${ }^{1,2}$ PANS is thought to be the result of several mechanisms and multiple etiologies, ranging from endocrine/metabolic causes to postinfectious autoimmune and neuroinflammatory disorders. ${ }^{3}$ Recently, a neuroimaging MRI investigation showed cerebral microstructural abnormalities in multiple brain structures, including basal ganglia, thalamus, and amygdala in children with PANS in comparison with healthy children. ${ }^{4}$

Sleep disorders represent one of the most frequent manifestations of PANS, involving around $80 \%$ of patients, often emerging at the onset of the psychiatric symptoms and subsequently regressing after the acute phase or following the course of the disease. ${ }^{5,6}$ Despite this frequent finding, to date few studies have been conducted analyzing the sleep characteristics of these patients, and only two polysomnographic studies have been published in patients diagnosed with PANS. ${ }^{7,8}$ Both studies report a higher prevalence of sleep disorders in PANS patients. The most common sleep disorders in patients with PANS are parasomnias (nightmares, nocturnal pavor, sleepwalking or somnambulism) as well as difficulties in falling asleep or maintaining sleep (early or intermediate insomnia), early awakenings (terminal insomnia), REM sleep disorders such as REM Sleep Without Atonia (RSWA), and sleep movement disorders such as Periodic Limb Movement Disorder (PLMD) are the most frequently mentioned. 8,9

The aim of the present study is to describe the clinical and polysomnographic features of sleep in a group of children diagnosed with PANS with no mention on medications for at least 4 weeks before enrollment. The main purpose is to identify putative relationships between sleep disorders and other PANS symptoms by using a data mining approach with fourth-generation Artificial Neural Networks, computational adaptive systems able to discover subtle trends and associations among variables. Starting from the analysis of these relationships, the authors intend to suggest an interwoven pathophysiological mechanism underling both sleep and PANS symptoms. The study also describes the condition known as "brain fog", a clouding of consciousness that affect the capacity to organize the thoughts, being wakeful and aware and pay attention $^{10}$ potentially linked to a plethora of possible causes including autoimmune disorders. For instance, people with Systemic Lupus Erythematosus experience a range of cognitive difficulties ("Lupus fog") such as confusion, difficulty in articulating thoughts and memory impairment. ${ }^{11}$ We suppose that this condition could affect also the children with PANS and that it could be related, at least in part, with the sleep abnormalities.

\section{Methods}

\section{Participants}

The participants are 23 children diagnosed with PANS during a time interval of six months (from March 1, 2019 to August 31, 2019), consecutively enrolled from the PANS/PANDAS outpatient-service at the Child and Adolescent Neuropsychiatry Unit, "A. Cao" Hospital "G. Brotzu" Hospital Trust, Cagliari. The sample included children from different Italian regions, being our service one of the few Italian centers specifically dedicated to PANS/PANDAS diagnosis and treatment. Inclusion criteria for their selection were diagnosis of PANS according to the criteria proposed by the 2015 Consensus Conference; ${ }^{5}$ drug-free period of at least 6 weeks; interruption of nutraceutical supplements (included Melatonin) at least 6 weeks before admission to the study.

All participants underwent a comprehensive clinical evaluation including a detailed interview aimed to assess sleepwake cycle and sleep disorders, cognitive assessment, blood chemistry examination (tests listed in Table 1), nasal and pharyngeal swab and polysomnography.

Beside PANS, specific DSM-5 disorders (Tic or Tourette Disorder, Obsessive-compulsive disorder, Mood Disorders, Anxiety Disorder, Attention Deficit Hyperactivity Disorder and Oppositional Defiant Disorder, Learning Disorders) were also considered and diagnosed by Kiddie SADS PL ${ }^{12}$ and specific assessment scales. 
Table I Laboratory Variables Measured in the Study Sample (PANS-Panel)

\begin{tabular}{|l|l|}
\hline $\begin{array}{l}\text { Routine blood } \\
\text { test }\end{array}$ & $\begin{array}{l}\text { Blood counts, sideraemia, Erythrocyte } \\
\text { sedimentation rate (ESR), C- Reactive Protein } \\
\text { (CRP), D-vitamin, Free Triiodothyronine (fT3), } \\
\text { Free thyroxine (fT4), Thyroid-stimulating } \\
\text { hormone (TSH), Neuron-specific Enolase (NSE) }\end{array}$ \\
\hline $\begin{array}{l}\text { Immunological } \\
\text { parameters }\end{array}$ & $\begin{array}{l}\text { Lymphocyte subpopulation, immunoglobulin and } \\
\text { complement profile, Anti-TSH Receptor } \\
\text { Antibodies (AbTR), Anti-thyroid peroxidase } \\
\text { (AbTPO), Anti-thyroglobulin Antibodies } \\
\text { (AbTG), Lupus Anti-Coagulant (LAC), Anti- } \\
\text { Nuclear Antibodies (ANA), anti-cardiolipin } \\
\text { Antibodies, Anti-Glicoprotein B2 Antibodies, } \\
\text { anti-deamidated gliadin, transglutaminase and } \\
\text { endomysial Antibodies. }\end{array}$ \\
\hline $\begin{array}{l}\text { Infectious } \\
\text { parameters }\end{array}$ & $\begin{array}{l}\text { Anti-streptolysin O (ASLO), Herpes Simplex } \\
\text { Virus I (HSV-I), Epstein Barr Virus (EBV), } \\
\text { Mycoplasma Pneumoniae and Chlamydia } \\
\text { Pneumoniae, Nasal and Pharyngeal swab. }\end{array}$ \\
\hline
\end{tabular}

In particular,

- Yale Global Tic Severity Scale (YGTSS) ${ }^{13}$ for the evaluation of tics (Score $\geq 20$ as threshold),

- Children's Yale-Brown Obsessive-Compulsive Scale $(\mathrm{CY}-\mathrm{BOCS})^{14}$ for the evaluation of obsessivecompulsive symptomatology (Score $\geq 7$ as threshold),

- Pediatric Acute-onset Neuropsychiatric Syndrome Scale $(\mathrm{PANSS})^{15}$ for the evaluation of specific PANS symptoms (Score $\geq 20$ as threshold with a possible maximum score of 54).

The level of impairment of global functioning was evaluated by Children's Global Assessment Scale (C-GAS); ${ }^{16}$ cognitive profile by Wechsler series scales (WISC-IV and WPPSI-III) considering Full Scale IQ, Verbal Comprehension Index (VCI), Perceptual Reasoning Index (PRI), Working Memory Index (WMI), Processing Speed Index (PSI); cognitive levels were dichotomized based on a cut-off of 85 (1 SD below the average).

\section{Polysomnography}

Each subject underwent a full-night ambulatory polysomnography (PSG) recording (Nox A1 - Nox Medical). The PSG was executed in the same week in which the clinical evaluation was performed. Sleep stages, respiratory and legs activity were scored manually by a clinical neurophysiologist expert in sleep medicine (PC), following standard AASM criteria ${ }^{17}$ (2018 update) on 30seconds epochs.

The following parameters were included in the polysomnographic study: EEG (six channels: one frontal, one central, and one occipital per hemisphere, referred to the contralateral earlobe), electrooculogram (electrodes placed $1 \mathrm{~cm}$ above the right cantus and $1 \mathrm{~cm}$ below the left cantus and referred to A1), electromyogram (EMG) of submental muscle, EMG of bilateral tibialis anterior muscle (bipolar derivations with two electrodes placed $3 \mathrm{~cm}$ apart on the belly of the anterior tibialis muscle of each leg), and one single-lead ECG. The sleep respiratory pattern was assessed by means of nasal airflow (nasal pressure cannula), thoracic and abdominal respiratory effort (strain gauge) and oxygen saturation (pulse-oximetry) during the study night. Sleep signals were stored on hard disk in European data format for further analysis. The polysomnographic evaluated parameters were Total Sleep Time (TST), Sleep Efficiency (SE), Sleep Latency (SL), REM Latency, N1\% TST, N2\% TST, N3\% TST, REM\% TST, Wake After Sleep Onset \% (WASO\%), Awakenings, Periodic Limb Movement Index (PLMI), RSWA (REM Sleep Without Atonia), and the presence of Frequent change position as index of restless sleep. ${ }^{18}$ The parameter "Ineffective Sleep" has been defined by values of Sleep Efficiency $<88 \%$; the parameter "Fragmented Sleep" has been defined by values of WASO $>10 \%$ and the diagnosis of Periodic Limb Movement Disorder (PLMD) has been formulated if the PLMI was $\geq 5.0$ events/hr in presence of a clinical sleep disturbance or daytime impairment. The diagnosis of pediatric obstructive sleep apnea syndrome (OSAS) was defined by an AHI $>1 / \mathrm{hr}$ and the presence of snoring or effortful, paradoxical, or obstructed breathing during the child' sleep, or in presence of sleepiness, hyperactivity, behavioral problems, or learning problems, according to ICSD-3 criteria for pediatric obstructive sleep apnea.

The parameter "Abnormal PSG" includes all the diagnosed sleep disorders (PLMD) and the altered PSG (Ineffective Sleep, Fragmented Sleep, and RSWA).

\section{Artificial Neural Networks Analysis}

For the analysis of the collected data, the Auto Contractive Map (Auto-CM) system has been used. Artificial Neural Networks (ANNs) are computational adaptive systems inspired by the functioning processes of the human brain: they are considered particularly useful to solve non-linear 
problems and to discover subtle trends and associations among variables. Based on their learning through an adaptive way (ie, extracting from the available data the information needed to achieve a specific aim and to generalize the acquired knowledge), the ANNs appear to be a powerful tool for data analysis in the presence of relatively small samples. ${ }^{19}$ The Auto Contractive Map (Auto$\mathrm{CM}$ ) system is a fourth generation unsupervised ANNs which has already been demonstrated to outperform several other unsupervised algorithms in a heterogeneous class of tasks. ${ }^{20}$ Auto-CM has been developed to explore the concomitant associations of different variables, and the potential relationships among variables in a multi-factor network relevant for the disease. The ultimate goal of this data mining model is to discover hidden trends and associations among variables since this algorithm is able to create a semantic connectivity map in which no linear associations are preserved, and explicit connection schemes are described.

Auto-CM requires a training phase necessary to learn how variables are interconnected. The learning algorithm of CM may be summarized in four orderly steps: a) Signal Transfer from the Input into the Hidden layer; b) Adaptation of the connections value between the Input layer and the Hidden layer; c) Signal Transfer from the Hidden layer into the Output layer; d) Adaptation of the connections value between the Hidden layer and the Output layer (see Supplementary Materials).

Based on the minimum spanning tree theory, the Auto$\mathrm{CM}$ reveals the connections among variables providing a graph in which the distances among variables reflect their bonding strength (weights). This approach provides the map of relevant connections between and among variables and the principal hubs of the system. Hubs can be defined as variables with the maximum amount of connections in the map. The Auto-CM does not pose randomly the initial weights. Conversely, the Auto-CM starts with the same value. Thus, the resulting graph is reproducible along many runs.

The MST represents what we could call the "nervous system" of any dataset. In fact, summing up all of the connection strengths among all the variables, we get the total energy of that system. The MST selects only the connections that minimize this energy, ie, the only ones that are necessary to keep the system coherent. Consequently, all the links included in the MST are fundamental, but, on the contrary, not every "fundamental" link of the dataset need be in the MST. Such limit is intrinsic to the nature of MST itself: every link that gives rise to a cycle into the graph (viz., that destroys the graph's 'treeness') is eliminated, whatever its strength and meaningfulness. To fix this shortcoming and to better capture the intrinsic complexity of a dataset, it is necessary to add more links to the MST, according to two criteria: (1) the new links have to be relevant from a quantitative point of view; (2) the new links have to be able to generate new cyclic regular microstructures, from a qualitative point of view. The additional links superimposed to MST graph generate a Maximally Regular Graph.

The Maximally Regular Graph (MRG) the graph whose hubness function attains the highest value among all the graphs generated by adding back to the original MST, one by one, the missing connections previously skipped during the computation of the MST itself. In other words, The MRG, generates, starting from the MST, the graph presenting the highest number of regular microstructures highlighting the most important connections of the dataset. The resulting "diamond" expresses the complexity core of the system and in our specific case, the core of the syndrome. The detailed description of Auto$\mathrm{CM}$ is provided in the Supplementary Material S1.

For statistical purposes, we converted some continuous variables in dichotomous measures. In particular, the ASLO patient's serum titers have been pooled in two groups (ASLO titer $>$ or $<$ of $200 \mathrm{IU} / \mathrm{mL}$ ), based on the upper reference limit. The ANA patient's serum titers have been pooled in two groups (titers $<$ or $>$ of 1: 120), based on the upper reference limit. ESR, NSE and CRP were evaluated as abnormal if above the upper reference limit of $15 \mathrm{~mm} / \mathrm{h}, 18 \mathrm{ug} / \mathrm{L}$ and $2.0 \mathrm{mg} / \mathrm{dl}$, respectively. Vitamin $\mathrm{D}$ was considered insufficient under the lower reference limit of $25 \mathrm{ng} / \mathrm{mL}^{21}$

\section{Ethical Approval}

This study was conducted in accordance with the Declaration of Helsinki and the Independent Ethical Committee of Cagliari University Hospital approved the study. All the parents were given a full explanation of the study methods and purposes and they gave their written consent.

\section{Results}

Between March 2019 and August 2019, a total of 43 individuals with PANS were screened for enrolment. Of these, $20 / 43(46.5 \%)$ were excluded as they did not meet inclusion criteria $(12 / 20$ were taking melatonin or vitamin D supplementation and $8 / 20$ were taking SSRI or 
Dopamine-receptors antagonist drugs). All enrolled children were drug free for any psychoactive and anti-inflammatory drugs and for dietary supplements for at least 6 weeks.

In the remaining 23 patients (19 males, 4 females; gender ratio of 5:1), mean age at enrollment was 9.8 years (SD: 2.6; range: 4.8-15.4), mean age at onset of PANS symptoms was 6.4 years (SD: 2.6; range: $2.5-$ 11.1), mean age at the time of diagnosis was 8.7 years (SD: 2.8; range: 3.8-15.1), corresponding to a mean diagnosis delay of 2.3 years (SD: 1.7; range: 0-6.5).

At the time of clinical assessment, 9/23 subjects $(39.1 \%)$ were in the acute phase of the disease (onset or relapsing phase), and the remaining 14/23 (60.8\%) had a chronic PANS presentation or were on the remitting phase of a "wax and wane" PANS course.

The descriptive characteristics of the sample are summarized in Table 2.

The clinical-anamnestic assessment about sleep-wake cycle obtained with the parental contribution showed that $14 / 23$ children had an urge to move the legs with associated unpleasant discomfort which, however, had no clear circadian rhythm, was present not solely at rest, and not always had benefit from the movements, preventing us to set RLS diagnosis. 10/23 children had snoring.

As expected, polysomnography showed abnormality in 17 out of $23(73.9 \%)$. In particular, in accordance with AASM criteria, 8/17 children (47\%) had an ineffective sleep, 10/17 (58.8\%) fragmented sleep, 11/17 (64.7\%) REM-Sleep Without Atonia (RSWA), and 8/17 (47.1\%) Periodic Limb Movement Disorder (PLMD). As suggested by the distribution of percentages, most patients had disordered sleep (“Abnormal PSG”). Similarly, among the19/23 patients diagnosed with Tic Disorder/Tourette Disorder, 8/19 (42.1\%) had PLMD, and 10/19 (52.6\%) showed RSWA polysomnographic features (Table 3).

The respiratory parameters were available only for $18 / 23(78.2 \%)$ patients, due to the difficulties for 5 children to accept the pulse-oximeter. Among them, $14 / 18(77.8 \%)$ subjects had a respiratory pattern characterized by snoring and 6/18 (33\%) had a condition of pediatric obstructive Sleep Apnea Syndrome (OSAS) with a severity score between mild (4/6) and severe (2/6) (see Table 3). These 2 patients with a severe OSAS underwent an adeno-tonsillectomy and reported an improvement in both OSAS (mean AHI 0,2 events/

Table 2 Demographic and Clinical Patients' Characteristics

\begin{tabular}{|c|c|c|c|}
\hline \multicolumn{4}{|l|}{ Continuous Variables } \\
\hline & Mean Value (Years) & Range (Years) & SD (Years) \\
\hline Mean age at study enrolment & 9.8 & $4.8-15.4$ & 2.6 \\
\hline Mean age at full symptom onset & 6.4 & $2.5-11.1$ & 2.6 \\
\hline Mean age at diagnosis & 8.7 & $3.8-15.1$ & 2.8 \\
\hline \multirow[t]{2}{*}{ Diagnostic mean delay } & 2.3 & $0-6.5$ & 1.7 \\
\hline & Mean Value & Range & SD \\
\hline Full Scale Intelligence Quotient (Full Scale IQ) & 94.6 & $55-116$ & 15.9 \\
\hline Verbal Comprehension Index (VCI) & 99.2 & $60-124$ & 18,9 \\
\hline Perceptual Reasoning Index (PRI) & 102.4 & $7 I-124$ & 15,5 \\
\hline Working Memory Index (WMI) & 90.5 & $70-118$ & 12,3 \\
\hline Processing Speed Index (PSI) & 84.6 & $65-109$ & 12,7 \\
\hline \multicolumn{4}{|c|}{ Discrete Variables } \\
\hline Gender & Females: 4/23 (I7\%) & Males: 19/23 (83\%) & \\
\hline Phase of disease & Acute: $9 / 23$ (39\%) & Chronic: 14/23 (61\%) & \\
\hline \multicolumn{4}{|c|}{ DSM-5 Associated Diagnosis } \\
\hline OCD I0/23 (43\%) & \multirow{3}{*}{\multicolumn{3}{|c|}{$\begin{array}{l}\text { ADHD/ODD: } 12 / 23(52 \%) \\
\text { Tic /TD: } 19 / 23 \text { (82\%) } \\
\text { Mood Disorders: } 2 / 23(8,6 \%)\end{array}$}} \\
\hline Anxiety $4 / 23(17 \%)$ & & & \\
\hline Learning Disorder 8/23 (34\%) & & & \\
\hline
\end{tabular}

Notes: Acute = symptoms onset or wax phase of a relapsing/remitting course; Chronic = Chronic course or wane phase of a relapsing/remitting course. Notably, both the Processing Speed Index (PSI) and the Working Memory Index (WMI) show a mean value relatively low, if compared to the other indices' scores.

Abbreviations: OCD, obsessive-compulsive disorder; ADHD, attention deficit hyperactivity disorder; ODD, oppositional defiant disorder. 
Table 3 Polysomnographic Parameters

\begin{tabular}{|c|c|c|c|c|c|}
\hline & \multirow[t]{2}{*}{ Mean } & \multirow[t]{2}{*}{ Std. Deviation } & \multirow[t]{2}{*}{ 95\% Confidence Interval } & \multicolumn{2}{|c|}{$\begin{array}{l}\text { D'Agostino \& Pearson Normality } \\
\text { Test }\end{array}$} \\
\hline & & & & $\mathbf{P}$ value & $($ Alpha $=0.05)$ \\
\hline Total sleep time. Min & 473.20 & 59.31 & $447.6-498.90$ & $0.8 I^{\mathrm{ns}}$ & Yes \\
\hline Sleep efficiency. \% & 89.57 & 5.86 & $87.04-92.11$ & $0.67^{\mathrm{ns}}$ & Yes \\
\hline Sleep Latency (min) & 14.53 & 12.79 & $8.995-20.06$ & $0.17^{\mathrm{ns}}$ & Yes \\
\hline REM latency (min) & 144.90 & 66.85 & $116-173.80$ & $0.74^{\mathrm{ns}}$ & Yes \\
\hline Sleep stage NI, \% & 7.94 & 5.47 & $5.574-10.30$ & $0.00 * *$ & No \\
\hline Sleep stage N2, \% & 46.69 & 6.19 & $44.01-49.36$ & $0.64^{\mathrm{ns}}$ & Yes \\
\hline Sleep stage N3, \% & 24.74 & 4.10 & $22.97-26.51$ & $0.53^{\mathrm{ns}}$ & Yes \\
\hline Sleep stage R, \% & 21.14 & 5.74 & $18.66-23.62$ & $0.72^{\mathrm{ns}}$ & Yes \\
\hline Wakefulness after sleep onset, \% & 10.16 & 5.43 & $7.813-12.51$ & $0.87^{\mathrm{ns}}$ & Yes \\
\hline WASO min & 50.87 & 29.84 & $37.97-63.77$ & $0.80^{\mathrm{ns}}$ & Yes \\
\hline Awakenings & 10.70 & 7.41 & $7.49-13.90$ & $0.05^{*}$ & No \\
\hline PLMS index, n/hour & 5.70 & 6.56 & $2.86-8.53$ & $<0.0001 * * * *$ & No \\
\hline Apnea-Hypopnea index, n/hour ${ }^{\#}$ & 2.79 & 4.56 & $0.45-5.14$ & $<0.0001 * * * *$ & No \\
\hline Oxygen Desaturation Index, n/hour ${ }^{\#}<3 \%$ & 2.01 & 2.84 & $0.55-3.47$ & $0.00 * * *$ & No \\
\hline SpO2mean $(\%)^{\#}$ & 96.31 & 0.57 & $96.01-96.60$ & $0.30^{\mathrm{ns}}$ & Yes \\
\hline $\mathrm{SpO} 2 \mathrm{~min}(\%)^{\#}$ & 92.13 & 1.54 & $91.3-92.95$ & $0.89^{\mathrm{ns}}$ & Yes \\
\hline Heart rate & 68.28 & 8.95 & $63.5 I-73.05$ & $0.07^{\mathrm{ns}}$ & Yes \\
\hline
\end{tabular}

Notes: ${ }^{\#}$ Respiratory parameters were available only for 18/23 patients, due to the difficulties for 5 children to accept the pulse-oximeter. Test for Gaussian distribution. GP: $0.1234\left(^{\mathrm{ns}}\right) ; 0.0332(*), 0.0021\left(^{* *}\right), 0.0002(* * *),<0.0001\left(^{* * * *}\right)$.

hour and mean ODI 0,2 events/hour) and PANS symptoms after surgery.

Biological parameters were out of the normal range in a large portion of patients. Serology and blood chemistry showed high antibody titers against Chlamydia Pneumoniae in $15 / 23$ participants $(65.2 \%)$ of which 10 / 15 were IgM and 5/15 IgG-mediated, and against Mycoplasma pneumoniae in $6 / 23$ participants $(26 \%)$ of which 4/6 were IgM and 2/6 IgG-mediated. Elevated ASLO (Anti Streptolysin-O; 11/23, 47.8\% of the sample) and Anti-Nuclear Antibodies (ANA) titers (5/23, 21.7\%) were also found. Neuron-specific enolase (NSE), an enzyme detected in serum following structural damage of neuronal brain cells, was elevated in $15 / 23$ subjects $(65.2 \%)$. Severe lower levels of vitamin D (14/23, $60.8 \%$ ) and of serum iron (sideraemia, 8/23, 34.7\%), were also detected. Erythrocyte Sedimentation Rate (ESR) faster-than-normal rate and a high level of C-Reactive Protein (CRP) were measured only in 4/23 $(17.3 \%)$ and $1 / 23(4.3 \%)$ patients.

With reference to the cognitive parameters, we found a discrepancy between the Processing Speed Index (PSI) (mean score 84.6) and the other intelligence scale mean score indexes (VCI 99.2; PRI 102.4; WMI 90.5). Since processing speed refers to the speed of cognitive processes and response output, this result accounts for a slower processing speed in the children of our sample.

\section{Semantic Connectivity Maps}

The semantic connectivity maps (Auto-CM method) Minimum spanning tree (MST) graphs show the strength of association across the clinical, neuropsychological, biological and polysomnographic variables visualized by the concept of "closeness": the variables whose connection weights are higher get relatively nearer and vice versa. The strength of the relation between variables increases progressively until the values of 1 (the strongest level of connection). In our maps, all the links strength values were above 0.8 (corresponding to a strong connection) and most of them were very close to 1 . As shown in all maps, the variable "Abnormal PSG", which includes all the diagnosed sleep disorders, acts as a hub having a considerable number of connections with the other clinical, neuropsychological and biological variables.

Map 1 shows the connections between all sleep parameters and the clinical variables, expressed by the standardized scores at the evaluation scales (YGTSS; CYBOCS; PANSS; C-GAS). The same map includes the diverse DSM-5 diagnoses of the disorders diagnosed in association to PANS. The "Maximally Regular Graph" 
(red diamond, for details see Supplementary Material S1) encompasses the variables with higher number of connections. These are represented by the DSM- 5 diagnosis of Tic disorder/Tourette disorder, the total YGTSS score $>20$, the PANSS total score $>20$, the CGAS $<60$, the "Abnormal PSG" and the presence of snoring (see Figure 1).

Map 2 explores the connections between all the sleep parameters and both the clinical dimensions assessed by PANSS and neuropsychological parameters assessed by Wechsler intelligence scales. This map is aimed to highlight the hidden connection between sleep characteristics and cognitive and behavioural symptoms. Both "Abnormal PSG" and "sleep disturbance" represent hubs with a high number of links. The "Maximally Regular Graph" (red diamond) includes also the motor symptoms, the learning cognitive symptoms, anxiety, irritability and OCD symptoms. Among PSG variables, the "Maximally Regular Graph includes snoring (see Figure 2).

Map 3 explores the connections between all sleep parameters and the laboratory variables exceeding the normal range. This Map reveals that the variable "Abnormal PSG" represents the hub with the highest number of links, receiving convergence from both sleep and biological parameters. The "Maximally Regular Graph" (red diamond) includes the D3 vitamin low level, the sideraemia low level, the positive anti-chlamydia antibodies and the ASLO high title as well as other clinical sleep variables (RSWA, PLMD, frequent position changes and Snoring) (see Figure 3).

\section{Discussion}

The results of the present study confirm sleep disturbances in children diagnosed with PANS, and strongly suggest that disordered sleep is a cardinal symptom of the PANS illness.

A growing scientific attention has recently focused on PANS. On one hand, several cohort studies demonstrated associations between different microbial infections and the abrupt emergence of psychiatric symptoms. On the other hand, biological studies disagree in showing PANS to have a clear immune basis and strong consistency for treatment with antimicrobials or immunotherapy is still lacking, with the only randomized, controlled clinical trial suggesting little outcome difference between placebo and immunotherapies. ${ }^{22}$ However, given the severe and sometimes disabling nature of PANS and the serious effects on family and child functioning, it is crucial to understand the biological basis of this syndrome and to distinguish this entity from others. The objective characterization of sleep alterations associated to PANS phenotype might be helpful in reaching this purpose.

\section{Polysomnographic Study}

Polysomnography was abnormal in $73.9 \%$ of our patients which is consistent with the finding of PANS consortium

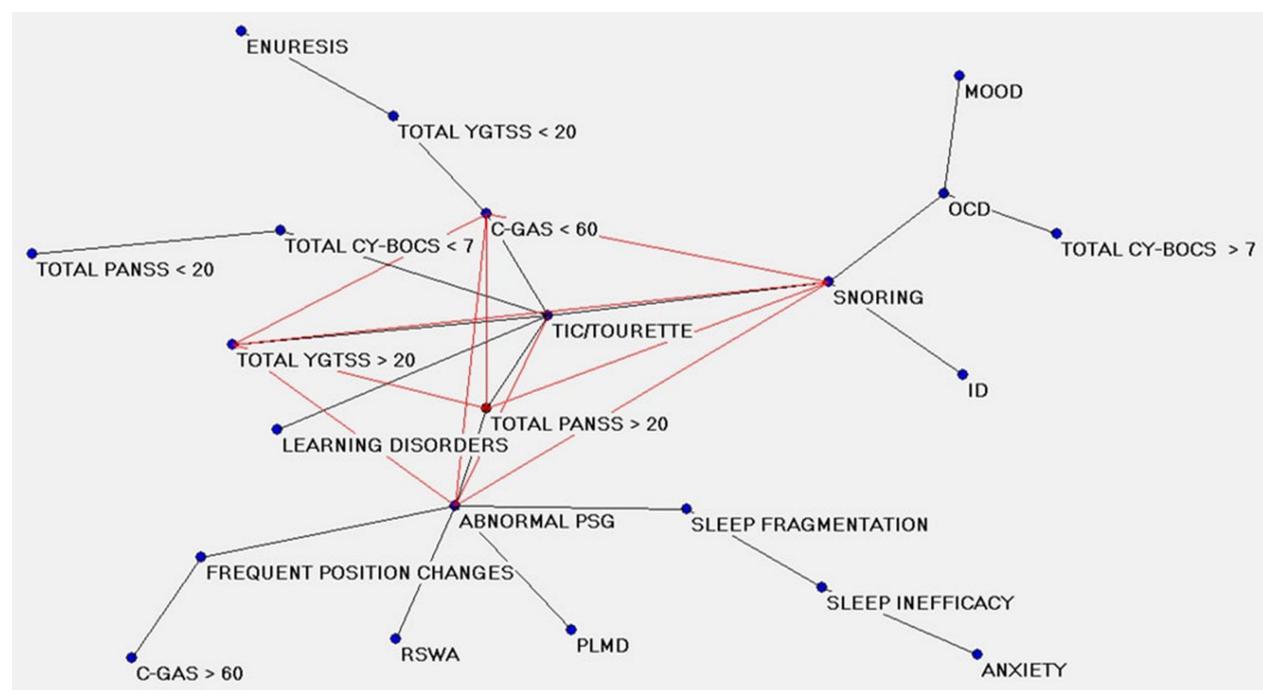

Figure I Map I. Connections between sleep parameters inferred by polysomnographic study, DSM-5 diagnoses, and all the behavioural parameters assessed by scales and checklists.

Abbreviations: PSG, polysomnography; OCD, obsessive-compulsive disorder; ID, intellectual disability; PLMD, periodic limb movement disorder; RSWA, REM sleep without atonia; Mood, mood disorders; Anxiety, anxiety disorders; Tic/Tourette, Tic/Tourette disorder; Total YGTSS, Total score in Yale Global Tic Severity Scale; Total CYBOCS, Total score in Children's Yale-Brown Obsessive-Compulsive Scale; Total PANSS, Total score in Pediatric Acute-onset Neuropsychiatric Syndrome Scale; C-GAS, score in Children's Global Assessment Scale. 


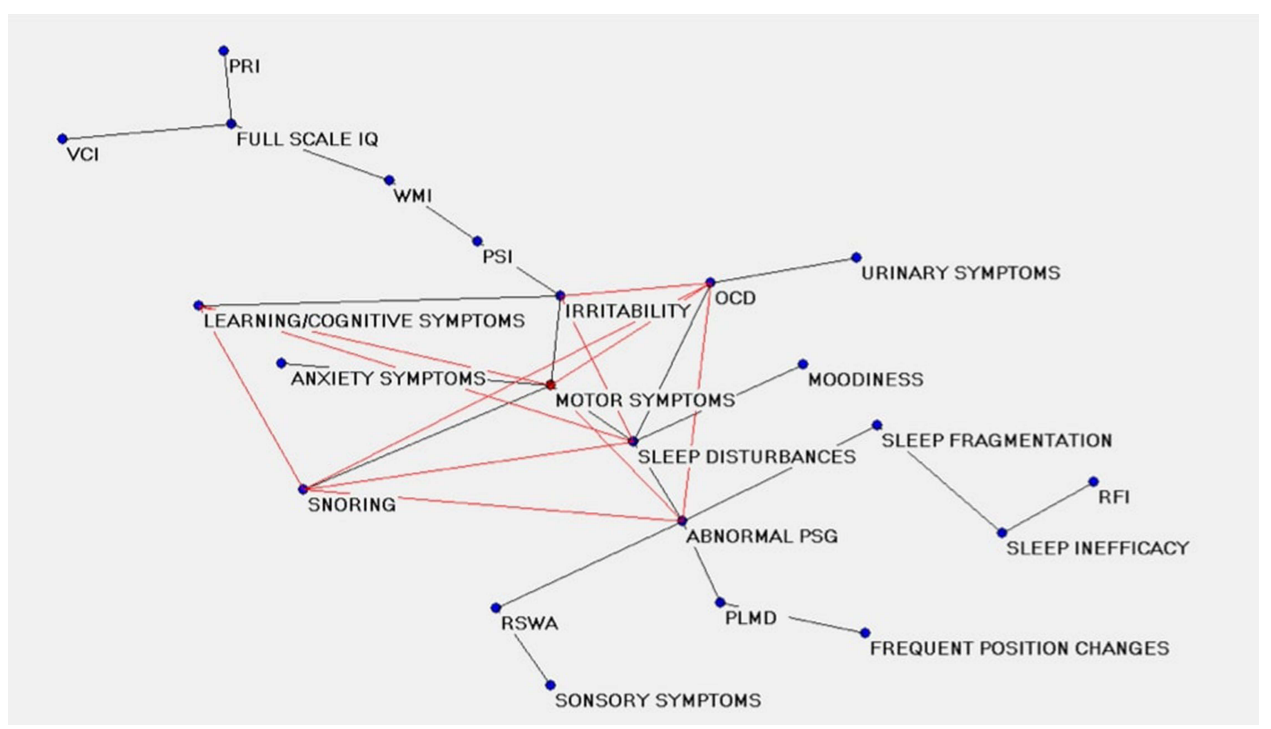

Figure 2 Map 2. Connections between sleep parameters inferred by polysomnographic study, clinical dimensions assed by PANSS and neuropsychological parameters assessed by Wechsler intelligence scales.

Abbreviations: PSG, polysomnography; PLMD, periodic limb movement disorder; RSWA, REM sleep without atonia; PRI, Perceptual Reasoning Index; VCI, Verbal Comprehension Index; PSI, Processing Speed Index; WMI, working memory index; Full-Scale IQ, Full-Scale Intelligence Quotient.

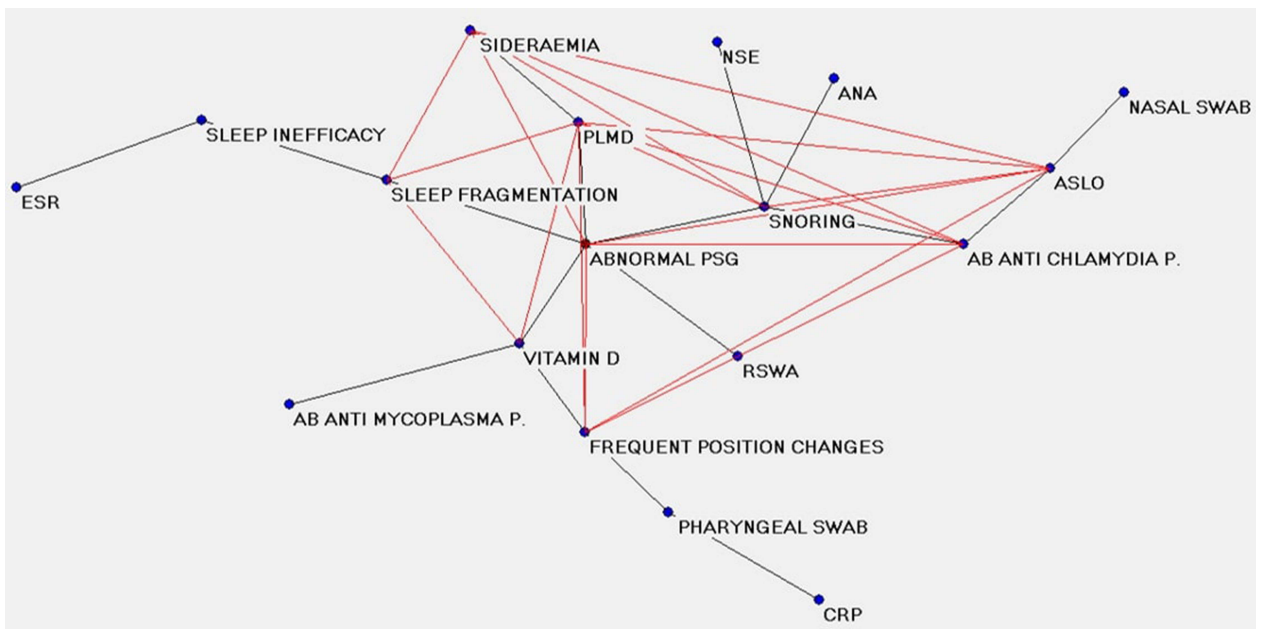

Figure 3 Map 3. Connections between clinical sleep parameters inferred by polysomnographic study and biological parameters which exceeded the normal range. Notes: Nasal and pharyngeal swab: culture positive for Group A beta-hemolytic streptococcus (GABHS) or Staphylococcus aureus (MRSA), Streptococcus pneumoniae (pneumococcus), Haemophilus influenzae or other upper respiratory tract pathogenic germs. The antistreptolysin O (ASLO) patient's values in the blood plasma have been pooled in two groups (antistreptolysin titer > or < of $200 \mathrm{IU} / \mathrm{mL}$ ), based on the upper reference limit. One or more IgG and IgM Antibodies against other germs (Anti Mycoplasma pneumoniae and Anti Chlamydia pneumoniae). The Antinuclear Antibodies (ANA) patient's values in the blood plasma have been pooled in two groups (titers < or > of I: 120), based on the upper reference limit. One or more inflammatory markers (erythrocyte sedimentation rate [ESR] and C-reactive protein [CRP]) above the upper reference limit.

Abbreviations: PSG, polysomnography; PLMD, periodic limb movement disorder; RSWA, REM sleep without atonia; ESR, Erythrocyte sedimentation rate; CRP, C-Reactive Protein; ANA, Anti-Nuclear Antibodies; NSE, Neuron-specific Enolase; ASLO, Anti Streptolysin O.

reporting sleep alterations in about $80 \%$ of patients with PANS both at the onset of symptoms and after the acute phase. $^{5}$

One of the main features of the present study is the complete pharmacological and nutraceutical wash-out condition of all enrolled patients. Compared to previous PANS polysomnographic studies, ${ }^{7,8}$ our study has no biases related to the potential drug-driven interferences on the sleep-wake rhythm and the intrinsic structure of sleep.

The subjects of our sample showed sleep disorders and disordered sleep both in acute and chronic phase of the disease, which may be linked to the new entity of 
restless sleep disorder described by DelRosso et al. ${ }^{18}$ In particular, PLMD and RSWA were largely represented. Notably, among the 19/23 patients diagnosed with Tic Disorder/Tourette Disorder, 10/19 (52.6\%) had a condition of RSWA and 8/19 (42.1\%) had a condition of PLMD. The prevalence rate of PLMD observed in the studied sample was significantly higher than that in the general pediatric population $(2-4 \%){ }^{24}$ The rates of RSWA were similar to those reported by Gaughan. ${ }^{7}$ Unfortunately, we could not compare our RSWA rate to that of the general pediatric population, since, to the best of our knowledge, this rate is not available in the existing literature. ${ }^{23}$ Moreover, the prevalence of PLMD in our Tic/Tourette's PANS patients was also significantly higher than that among pediatric patients with isolated Tourette's Disorder $(6 \%$ described by KostaneckaEndress et al). ${ }^{25}$ This difference might represent a possible distinction-key between a "pure" Tourette's Disorder and a Tourette's Disorder diagnosed in the context of a PANS.

The high prevalence of PLMD among PANS patients may suggest a specific involvement of dopaminergic circuits, in the disorder as PLMD is thought to be sustained by a deficit of motor inhibition during sleep. ${ }^{26-30}$ Periodic Limb Movements (PLMs) are associated with a hypo function of dopaminergic system as suggested by their suppression after treatment with L-dopa or dopaminergic agonist drugs, whereas they may be induced or worsened by dopamine-receptor antagonists. ${ }^{31}$

In patients with PLMs in sleep or restless legs syndrome, PET and SPECT studies have shown a mild presynaptic nigrostriatal and postsynaptic striatal dopaminergic hypofunction. ${ }^{32}$

In patients with Parkinson's disease reduced striatal [(123)I] beta-CIT binding correlated with the number of periodic limb movements in sleep, ${ }^{33,34}$ Parkinsonian patients off of dopaminergic treatment had significantly higher rates of PLMs than patients under treatment. ${ }^{35}$

\section{Semantic Connectivity Maps}

Artificial Neural Network methodology and the AutoContractive Map was used to investigate the putative relationships among the frequent PSG abnormalities and clinical, neuropsychological and laboratory parameters. Exploiting all not obvious links among the full spectrum of variables, the Auto-Contractive Map method revealed the simultaneous connections among them, facing the complexity of PANS phenotype.
The semantic connectivity Map 1 (Figure 1) shows the connections between sleep parameters, DSM-5 diagnoses, and all the behavioral parameters assessed by scales and checklists. The clearest evidence of this map is that the variable "Abnormal PSG" is related to higher PANS total score, suggesting a strong impact of sleep disturbance on PANS phenotype. Interestingly, "Tic/Tourette" diagnosis is the variable closer to the "Abnormal PSG", confirming the strong connection between sleep abnormalities and the presence of daily uncontrolled movement as tics. An extensive nationwide population-based study showed as Tourette Disorder per se is an independent risk factor for sleep disorders in children with associated neurodevelopmental disorders. ${ }^{36}$ However, the overall incidence rate of sleep disorders in TD subjects reported in Lee's study ${ }^{36}$ (7.24\%) is largely lower than the incidence rate of sleep disorders in our PANS series, suggesting a wider involvement of the sleep regulation mechanisms in PANS than in TD.

The "Maximally Regular Graph" (red diamond) encompassing the variables with higher number of connections, also includes the variable "snoring". This is an interesting finding since snoring is usually related in children with adenotonsillar hypertrophy, which, in turn, represents a potential cause of OSAS. ${ }^{37}$ In our sample $33 \%$ of the 18 patients for which oximeter data were available had OSAS. A previous study, aimed to describe the otorhinolaryngologic findings in patients with Pediatric Autoimmune Neuropsychiatric Disorders Associated with Streptococcal Infections (PANDAS), found a high rate (65.7\%) of adenoid and tonsil hypertrophy. ${ }^{38}$ The authors stated that not all children with adenotonsillar hypertrophy suffer also from sleep breathing disorders and that the correlation between the degree of lymphatic structures hypertrophy and the severity of obstructive respiratory symptoms is not linear. However, the same authors discuss the efficacy of adenotonsillectomy for pediatric OSAS and, according to the guidelines for tonsillectomy (American Academy of Otolaryngology-Head \& Neck Surgery) ${ }^{39}$ suggest to consider the surgical therapeutic option also for PANDAS/PANS patients.

The semantic connectivity Map 2 (Figure 2) shows the connections between sleep parameters, clinical dimensions assessed by PANSS and neuropsychological parameters assessed by Wechsler intelligence scales. "Abnormal PSG" and "sleep disturbances" variables represent hubs with a high interconnectivity with motor symptoms, learning cognitive symptoms, anxiety, irritability and OCD. 
Therefore, the "Maximally Regular Graph" (red diamond) includes both cognitive and psychiatric symptoms strictly linked to sleep disturbances, suggesting a central role of these last in defining the PANS phenotype. Among PSG variables, snoring is the closest to learning and cognitive dimension of PANS. A potential impact of sleep disordered breathing symptoms on daily cognitive performances has already been described by many authors. ${ }^{40,41}$ A similar impairment of both sleep and cognitive aspects also occurs in other sleep disturbances such as narcolepsy and Kleine-Levin syndrome. ${ }^{42}$ In these conditions, sleep alterations are often associated with a certain degree of cognitive dysfunction as confusion and fogging. ${ }^{43}$

Interestingly, the semantic connectivity Map 2 (Figure 2) shows a close link between the sleep variables, encompassed in the red diamond, and the cognitive indices "Processing Speed Index (PSI)" and "Working Memory Index (WMI)" of the Wechsler intelligence scales for children. Processing speed is thought to be the ability to rapidly process novel information and to identify, discriminate, integrate, make a decision about information while working memory accounts for higher order thinking processes and, in particular, for the ability to temporarily store and manipulate new information to ensure successful task execution. ${ }^{12}$ We can assume that both these functions are impaired in the condition known as "brain fog" described as a weakening in memory, attention, executive function, and the speed of cognitive processing. This condition has been clinically observed in a large part of the children of our sample and confirmed by the result of the cognitive test, showing relatively low mean scores in PSI and WMI index (84.6 and 90.5, respectively). Meaningfully, reduced speed of complex information processing is the impairment most consistently found in chronic fatigue syndrome $(\mathrm{CFS})^{44}$ a disease strongly associated with a brain fog condition. A more recent study conduct in adolescents with $\mathrm{CFS}^{45}$ found that IQ scores tested lower than the IQ scores of healthy peers with an equivalent school level suggesting that the lower IQ outcomes could be due to concentration problems based on a lowered processing speed. At the same time, the "brain fog" condition is largely described among different autoimmune and inflammatory diseases. We mentioned in the introduction the Systemic Lupus Erythematosus disease as a condition that can lead to experience a range of cognitive difficulties ("Lupus fog") such as confusion, difficulty in articulating thoughts and memory impairment. ${ }^{11}$ A mild degradation of cognitive functions, referred to as "brain fog", has been also reported in patients with coeliac disease. ${ }^{46}$ Such deficits associated with sleep disturbance, also occur in patients with Crohn's disease, particularly in association with systemic inflammatory activity. ${ }^{47}$ Taken together, these data could indicate a possible link between sleep disorders/disordered sleep and brain fog and the association of both these conditions with systemic inflammation. Furthermore, the symptomatic overlap of PANS with other autoimmune diseases and other sleep disorders of wellknown immune-mediated pathogenesis is consistent with the explanation of PANS as a complex syndrome encompassing different symptoms, epiphenomena of a common inflammatory/autoimmune condition.

The analysis of the connections between sleep and biological parameters by semantic connectivity Map 3 (Figure 3), shows that the node "Abnormal PSG" acts as a hub, receiving a great number of convergences. This evidence stresses, once again, the central role of sleep disturbances and disordered sleep in PANS phenotype. The "Maximally Regular Graph" (red diamond) links the sleep variables (PLMD, Sleep fragmentation, RSWA, frequent position changes and Snoring) with some metabolic and infective variables linked to PANS. In particular, some infectious markers such as a high ASLO titer and the presence of anti-Chlamydia antibodies, appears closely linked to sleep variables suggesting a shared dysimmune basis, possibly triggered by an infectious episode. This concept has already been proposed by some authors which have stressed the bidirectional relationship between sleep and immunity against infections, starting from the evidence of a large proportion of patients with narcolepsy having antibodies (ASLO) against Group A Beta-Hemolytic Streptococcal (GABHS). ${ }^{48,49}$ The authors' hypothesis was that this bacterium might trigger narcolepsy through an autoimmune mechanism. Furthermore, starting from the observation of a high density of cytokines' receptors (particularly interleukins' receptors) in hypothalamic neurons, some authors propose that the wake-sleep rhythm and immune system modulate each-other. ${ }^{48}$ However, not only GABHS but also other bacterial pathogens frequently causing sore throat (eg, Chlamydia pneumoniae and Mycoplasma pneumoniae) are supposed to be triggers for PANS symptoms ${ }^{5}$ and, specifically for sleep disorders related to PANS, as suggested by the strong link between the anti-Chlamydia and anti-Mycoplasma antibodies and sleep variables, in our sample.

As specific marker of neuronal injury we found increased serum level of Neuron Specific Enolase (NSE) 
in a large portion of our sample. NSE is a glycolytic enzyme found in neuronal and neuroendocrine tissues, associated with nonspecific central nervous system injury, such as cerebral vascular accident, brain metastasis, systemic sclerosis, stroke, anoxic encephalopathy and encephalitis. $^{50}$ Interestingly, increased serum levels of NSE were found also in Acute Sleep Deprivation ${ }^{51}$ and chronic insomnia disorder. ${ }^{52}$ As the semantic connectivity map showed, NSE serum level is closely related to sleep parameters in our PANS sample. This link could be indicative for either neuronal damage, impaired sleep, or both.

A second significant correlation described by the semantic connectivity Map 3 (Figure 3) is the link between sleep parameters and the condition of hypo-sideraemia (low iron serum level). Systemic iron deficiency is described in about two-thirds of children with restless legs syndrome (RLS) and it seems to be related to a more severe symptomatology. ${ }^{53,54}$ In the Wisconsin Sleep Cohort, a PLM index $>15$ was associated with low $(<50 \mathrm{ng} / \mathrm{mL})$ serum ferritin levels suggesting a role for low iron stores in the pathogenesis of Periodic Limb Movements. ${ }^{26}$ A current explanation is that iron deficiency may result in a dopaminergic dysfunction supporting the RLS and PLMD, since iron is a necessary cofactor for dopamine production via tyrosine hydroxylase. ${ }^{55}$ Iron deficiency adversely affects the basal ganglia function potentially leading to cognitive and motor dysfunctions, condition which could represent an intersection between PANS, Tourette Disease and PLMD. ${ }^{56}$

Finally, in Map 3 (Figure 3) we observed a strong connection between vitamin D deficiency and "Abnormal PSG". This is a somehow expected result given the role of vitamin $\mathrm{D}$ both as immunomodulatory agent and fundamental factor for a balanced neurotransmitter setting in the central nervous system. ${ }^{24}$ A recent meta-analysis indicates that serum $25(\mathrm{OH})$ D $<20 \mathrm{ng} / \mathrm{mL}$ could significantly increase the risk of unhealthy sleep and sleep disorders. ${ }^{57}$ At the same time, a large amount of literature supports the central role of vitamin $\mathrm{D}$ in modulating immunological functions. ${ }^{58}$ Insufficient vitamin D levels are thought to be linked to a higher susceptibility for infectious and autoimmune diseases. ${ }^{59-61}$ Within this framework the connection between sleep disorder, vitamin $\mathrm{D}$ deficiency and PANS is an expected condition.

\section{A Hypothetical Dysfunctional Model}

Obsessive-compulsive, tic and sleep disorders have in common varying degrees of dysfunction in attentional vigilance and motor disturbances. Starting from this consideration, we propose a hypothetical dysfunctional model explaining the cooccurrence of sleep, motor, obsessive-compulsive and cognitive symptoms in PANS, graphically explained in Figure 4.

Basal ganglia play a crucial role in sleep physiology and pathophysiology. ${ }^{62}$ The midbrain dopamine system via the dorsal and ventral striatum tunes cortical inputs, regulating a wide range of functions including cognition, motor behavior, and sleep-wake states. ${ }^{63-65}$ Recent evidence from sleep animal models indicate that stimulation of nigrostriatal dopaminergic pathways, activating the external globus pallidus (GPe) increases sleep and EEG delta power, while stimulation of mesolimbic dopaminergic, activating the nucleus accumbens, induces arousal, decreasing sleep and reducing EEG power. ${ }^{66,67}$

Recent neuroimaging studies indicate that microstructural differences (ie, apparent diffusion coefficient, but not regional brain volume nor cerebral blood flow) may be observed in the basal ganglia, thalamus, and amygdala of children and adolescents with PANS. ${ }^{4}$ Interestingly, recent evidence indicates that also thalamus and amygdala play an important role in sleep regulation. ${ }^{68}$ Considering the complex microstructure of the basal ganglia, ${ }^{69}$ PANS neuro-inflammation may heterogeneously impact specific sub regions modulated by midbrain dopaminergic pathways, leading to apparently opposite symptoms (ie tics and PLMD). Interestingly, high prevalence of a PLMD (a hypo-dopaminergic condition) in patient also showing motor and vocal tics (a hyper-dopaminergic condition) may appear contradictory and of puzzling explanation: in fact, dopaminergic regulation of sleep-wake cycle may be of help.

The thalamus and basal ganglia interconnect distant parts of the cerebral cortex via cortico-thalamo-cortical and cortico-striato-thalamic loops. In recent years the parallel and segregated model of basal ganglia modulation of cortical motor, cognitive and limbic function ${ }^{70}$ evolved into a model of multiple spatially distant cortical projections to the subcortex, describing the circuits as "parallel and integrative" rather than purely parallel. ${ }^{71}$ This integrative model may explain why even small lesions in the thalamus or basal ganglia can be neurologically remarkable, whilst similarly sized lesions in the cerebral cortex may go unnoticed. ${ }^{72,73}$ Immune-mediated impairment of basal ganglia may explain the specific cognitive relative impairment of cognition (WISC IV Processing Speed Index and, more in general "Brain fog") observed in our PANS patients and other case series. 


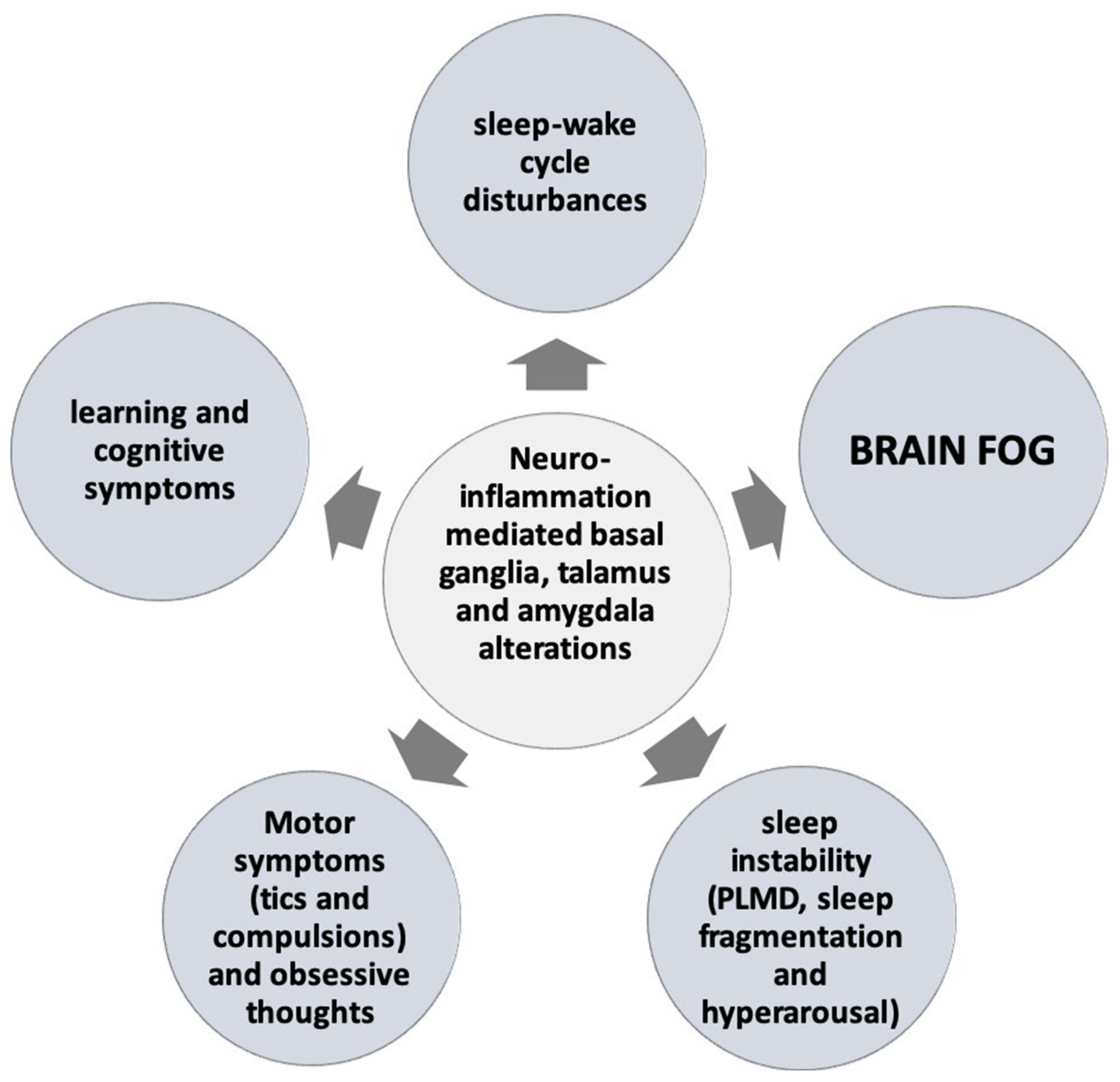

Figure 4 A putative dysfunctional model explaining the co-occurrence of sleep, motor and cognitive symptoms in PANS: PANS-related obsessive-compulsive and tic symptoms, putatively following insults to the basal ganglia and to the consequently functional alterations of CSTC motor circuit. Inflammatory-driven alteration of basal ganglia may explain the reduction of the mental capacity to concentrate or to think or reason clearly ("Brain fog") observed in PANS patients and, at the same time, may favour the sleep instability and sleep-wake cycle disturbances.

\section{Limitations}

Lack of a control group, as only PANS patients were included, could be the major limitation of our study together with the relatively small sample size. Nevertheless, our chosen statistical model allowed us to analyze a single group data set. Differently from the classical statistical tests, analytical methods based on Artificial neural networks may manage complexity even with relatively small samples and with a subsequent unbalanced ratio between variables and records. In this connection, it is important to note that adaptive learning algorithms of inference, based on the principle of a functional estimation like artificial neural networks, overcome the problem of dimensionality.

Comparison with typical neurodevelopmental subjects and/or with patients presenting a limited association of PANS criteria, but not the complete disorder, may help to verify the strength of the observed associations.
Furthermore, an enlarged sample size will be useful to confirm the present results. Further studies including more subjects and a control cohort are warranted to confirm these findings, leading to the inclusion of sleep abnormalities among PANS major diagnostic criteria.

Another limitation of this study was that a lab attended video-PSG recording was not performed, and consequently an objective visual information about the night of the recording is missing, thus in order to report any abnormal behavior during sleep, parents were asked to supervise children during the recording.

A further limitation of our study is the lack of female in our sample. Even though, according to the study of Calaprice and coll, ${ }^{74}$ the gender ratio in PANS is estimated to be 1.8 males to 1 female, the consecutive patients enrolled in the present study were mainly males (gender ratio $5: 1$ ). The possibility that this circumstance affected the results is purely speculative, but it would be 
appropriate to study the sleep characteristics in clinical samples including both genders.

Finally, the iron deficiency found in our sample have not been discussed in light of the ferritin levels because the lack of ferritin measurement. Since both RLS and/or PLMD are more closely linked to ferritin levels, further studies should provide the whole analysis of iron metabolism parameters.

\section{Conclusion}

Taken together, altered sleep has been linked to abnormalities in basal ganglia and neuroinflammatory diseases (ie, narcolepsy), and seeing that a recent study shows microstructural changes to the deep grey matter in patients with PANS, ${ }^{4}$ it is not surprising that this PANS cohort has sleep disturbances.

Several lines of evidence are consistent with this argument:

- the symptomatic overlap with other sleep disorders (narcolepsy and Klein-Levine syndrome) of established immune-mediated pathogenesis;

- the strong link between some infectious markers (antiChlamydia Pneumoniae and ASLO titers) and sleep disorders, supporting the infective trigger causative model via abnormal activation of the immune system;

- the association between a deficit of vitamin D, a known immunomodulatory agent, and the polysomnographic alterations;

- the association with PLMD and with serum iron deficiency suggesting an impairment of dopaminergic system in the pathogenesis of both PLM and tics in PANS.

Nevertheless, our sample size and the limitation of the present study design does not allow firm conclusion on the pathophysiology of PANS, even the present results may be considered a conceptual framework useful for organizing further empirical research.

A complete evaluation of sleep should be performed for all PANS patients since the onset of the disorder, and a polysomnographic investigation should be suggested in all PANS patients for whom the parents report sleep disturbance as well as for patients with strong indicators of cognitive alterations, as brain fog condition, daily sleepiness, confusion or attentive difficulties.

In conclusion, the present study underlines that sleep alterations represent, for prevalence and impact on quality of life, a cardinal symptom of PANS phenotype. Currently, sleep abnormality is not a major criterion for this diagnosis. Considering that measuring sleep abnormalities maybe more objective than measuring psychiatric symptoms, clinicians and researchers should consider revising the diagnostic criteria and tracking methods to include sleep quality.

\section{Abbreviations}

AASM, American Academy of Sleep Medicine; ADHD, attention deficit hyperactivity disorder; AHI, Apnea Hypopnea Index; ANA, Anti-nuclear Antibodies titer; ANNs, Artificial Neural Networks; ASLO, Anti Streptolysine titer; Auto-CM, Auto Contractive Map; C-GAS, Children's Global Assessment Scale; CRP, C-Reactive Protein; CFS, Chronic Fatigue Syndrome; CYBOCS, Children's Yale-Brown Obsessive-Compulsive Scale; DSM-5, The Diagnostic and Statistical Manual of Mental Disorders - 5th edition; ECG, Electrocardiogram; EEG, Electroencephalogram; EMG, Electromyogram; ESR, Erythrocyte Sedimentation Rate; GABHS, Group A BetaHemolytic Streptococcal; GPe, external Globus Pallidus; Kiddie SADS PL, The Kiddie Schedule for Affective Disorders and Schizophrenia Present and Lifetime Version; L-dopa, Levodopa; IQ, Intelligence Quotient; MRI, Magnetic Resonance Imaging; MST, Minimum Spanning Tree; N1, N1 phase of NREM sleep; N2, N2 phase of NREM sleep; N3, N3 phase of NREM sleep; NREM, not rapid eye movement sleep stage; NSE, Neuron-specific enolase; OCD, ObsessiveCompulsive Disorder; ODD, Oppositional Defiant Disorder; OSAS, Obstructive Sleep Apnea Syndrome; PANDAS, pediatric autoimmune neuropsychiatric disorders associated with streptococcal infections; PANS, Pediatric Acute-onset Neuropsychiatric Syndrome; PANSS, Pediatric Acute-onset Neuropsychiatric Syndrome Scale; PET, Positron-Emission Tomography; PLMD, Periodic Limb Movement Disorder; PLMI, Periodic Limb Movement Index; PLMs, Periodic Limb Movements; PSG, Polysomnography; PRI, Perceptual Reasoning Index; PSI, Processing Speed Index; RBD, REM Behavior Disorder; REM, Rapid Eye Movement Sleep stage; RSWA, REM Sleep Without Atonia; SD, Standard Deviation; SE, Sleep Efficiency; SL, Sleep Latency; SPECT, Single Photon Emission Computed Tomography; SSRI, Selective Serotonin Reuptake Inhibitors; TD, Tourette Disorder; TST, Total Sleep Time; VCI, Verbal Comprehension Index; WASO, Wake After Sleep Onset; WISH-IV, Wechsler Intelligence Scale for Children - 4th edition; WMI, Working Memory Index; WPPSI-III, Wechsler Preschool and Primary Scale of Intelligence - 3rd edition; YGTSS, Yale Global Tic Severity 
Scale; [(123)I]beta-CIT, Iodine-123-beta-carbomethoxy-3 beta-(4-iodophenyltropane) (CIT).

\section{Acknowledgments}

We thank Mrs Carmen Garau, Mrs Josè Garau, Mr Alessandro Ledda \& Mr Fabrizio Genugu for technical support on sleep recording assessments. This work was performed at the Sleep Disorder Center, University of Cagliari and at the Outpatient-service at the Child and Adolescent Neuropsychiatry Unit, "A. Cao" Hospital "G. Brotzu" Hospital Trust, Cagliari. These authors share senior authorship: Enzo Grossi and Alessandro Zuddas.

\section{Disclosure}

Alessandro Zuddas reports grant and research support from Angelini, Jansenn, Lundbeck, Otsuka, Roche, Sevier, the European Union and the Sardinian Regional Health Secretary; and honoraria or consultation fees from Angelini, Edupharma, Servier, and Takeda. None of them were related to the submitted work. The authors reported no other potential conflicts of interest for this work.

\section{References}

1. Swedo SE. Pediatric autoimmune neuropsychiatric disorders associated with streptococcal infections (PANDAS). Mol Psychiatry. 2002;7(S2):24-25. doi:10.1038/sj.mp.4001170

2. Thienemann M, Murphy T, Leckman J, et al. Clinical management of pediatric acute-onset neuropsychiatric syndrome: part I - psychiatric and behavioral interventions. $J$ Child Adolesc Psychopharmacol. 2017;27(7):566-573. doi:10.1089/cap.2016.0145

3. Frankovich J, Thienemann M, Pearlstein J, Crable A, Brown K, Chang K. Multidisciplinary clinic dedicated to treating youth with pediatric acute-onset neuropsychiatric syndrome: presenting characteristics of the first 47 consecutive patients. $J$ Child Adolesc Psychopharmacol. 2015;25(1):38-47. doi:10.1089/cap.2014.0081

4. Zheng J, Frankovich J, McKenna ES, et al. Association of Pediatric Acute-Onset Neuropsychiatric Syndrome with microstructural differences in brain regions detected via diffusion-weighted magnetic resonance imaging. JAMA Netw open. 2020;3(5):e204063. doi:10.1001/ jamanetworkopen.2020.4063

5. Chang K, Frankovich J, Cooperstock M, et al. Clinical evaluation of youth with pediatric acute-onset neuropsychiatric syndrome (PANS): recommendations from the 2013 PANS consensus conference. J Child Adolesc Psychopharmacol. 2015;25(1):3-13. doi:10.1089/cap.2014.0084

6. Gagliano A, Galati $C$, Ingrassia $M$, et al. Pediatric acute-onset neuropsychiatric syndrome: a data mining approach to a very specific constellation of clinical variables. J Child Adolesc Psychopharmacol. 2020;XX(Xx):1-17. DOI:10.1089/cap.2019.0165

7. Gaughan T, Buckley A, Hommer R, et al. Rapid eye movement sleep abnormalities in children with Pediatric Acute-Onset Neuropsychiatric Syndrome (PANS). J Clin Sleep Med. 2016;12(7):1027-1032. doi: $10.5664 /$ jesm. 5942

8. Santoro JD, Frankovich J, Bhargava S. Continued presence of period limb movements during REM sleep in patients with chronic static Pediatric Acute-Onset Neuropsychiatric Syndrome (PANS). J Clin Sleep Med. 2018;14(7):1187-1192. doi:10.5664/jcsm.7222
9. Swedo E. from research subgroup to clinical syndrome: modifying the PANDAS criteria to describe PANS (Pediatric Acute-onset Neuropsychiatric Syndrome). Pediatr Ther. 2012;02(02). doi:10.41 72/2161-0665.1000113

10. Schildkrout B. Unmasking Psychological Symptoms: How Therapists Can Learn to Recognize the Psychological Presentation of Medical Disorders. John Wiley \& Sons; 2011.

11. Mackay M. Lupus brain fog: a biologic perspective on cognitive impairment, depression, and fatigue in systemic lupus erythematosus. Immunol Res. 2015;63(1-3):26-37. doi:10.1007/s12026-015-8716-3

12. Kaufman AS, Flanagan DP, Alfonso VC, Mascolo JT. test review: wechsler intelligence scale for children, fourth edition (WISC-IV). JPsychoeduc Assess. 2006;24(3):278-295. doi:10.1177/0734282906288389

13. Leckman JF, Riddle MA, Hardin MT, et al. The Yale Global Tic Severity Scale: initial testing of a clinician-rated scale of tic severity. J Am Acad Child Adolesc Psychiatry. 1989;28(4):566-573. doi:10. 1097/00004583-198907000-00015

14. Scahill L, Riddle MA, McSwiggin-Hardin M, et al. Children's YaleBrown Obsessive Compulsive Scale: reliability and validity. $J \mathrm{Am}$ Acad Child Adolesc Psychiatry. 1997;36(6):844-852. doi:10.1097/ 00004583-199706000-00023

15. Murphy TK, Gerardi DM, Leckman JF. Pediatric acute-onset neuropsychiatric syndrome. Psychiatr Clin North Am. 2014;37(3):353-374. doi:10.1016/j.psc.2014.06.001

16. Shaffer D, Gould MS, Brasic J, et al. A children's global assessment scale (CGAS). Arch Gen Psychiatry. 1983;40(11):1228-1231. doi:10.1001/archpsyc.1983.01790100074010

17. Berry RB, Brooks R, Gamaldo C, et al. AASM scoring manual updates for 2017 (version 2.4). J Clin Sleep Med. 2017;13 (5):665-666. doi:10.5664/jesm.6576

18. DelRosso LM, Picchietti DL, Spruyt K, et al. Restless sleep in children: a systematic review. Sleep Med Rev. 2020;56:101406. doi:10.1016/j.smrv.2020.101406

19. Buscema M, Grossi E, Montanini L, Street ME, Zenclussen AC. Data mining of determinants of intrauterine growth retardation revisited using novel algorithms generating semantic maps and prototypical discriminating variable profiles. PLoS One. 2015;10(7):e0126020. doi:10.1371/journal.pone.0126020

20. Buscema M, Sacco PL. Digging deeper on "deep" learning: a computational ecology approach. Behav Brain Sci. 2017;40:e256. doi:10.1017/S0140525X1700005X

21. Vierucci F, Del Pistoia M, Fanos M, Erba P, Saggese G. Prevalence of hypovitaminosis D and predictors of vitamin D status in Italian healthy adolescents. Ital J Pediatr. 2014;40(1):54. doi:10.1186/18247288-40-54

22. Wilbur C, Bitnun A, Kronenberg S, et al. PANDAS/PANS in childhood: controversies and evidence. Paediatr Child Health. 2019;24 (2):85-91. doi:10.1093/pch/pxy145

23. Shukla G, Gupta A, Chakravarty K, et al. Rapid Eye Movement (REM) sleep behavior disorder and REM sleep without atonia in the young. Can J Neurol Sci. 2019;1-9. doi:10.1017/cjn.20 19.302 .

24. Rulong G, Dye T, Simakajornboon N. Pharmacological management of restless legs syndrome and periodic limb movement disorder in children. Pediatr Drugs. 2018;20(1):9-17. doi:10.1007/s40272-0170262-0

25. Kostanecka-Endress T, Banaschewski T, Kinkelbur J, et al. Disturbed sleep in children with Tourette syndrome A polysomnographic study. J Psychosom Res. 2003;55(1):23-29. doi:10.1016/S0022-3999(02) 00602-5

26. Hamilton-Stubbs P, Walters AS. Periodic limb movements. Encycl Mov Disord. 2010;(Coleman 1980):444-449. doi:10.1016/B9780-12-374105-9.00132-5

27. Manconi M, Ferri R, Zucconi M, et al. First night efficacy of pramipexole in restless legs syndrome and periodic leg movements. Sleep Med. 2007;8(5):491-497. doi:10.1016/j.sleep.2006.10.008 
28. Turjanski N, Lees AJ, Brooks DJ. Striatal dopaminergic function in restless legs syndrome: 18F-dopa and 11C-raclopride PET studies. Neurology. 1999;52(5):932. doi:10.1212/WNL.52.5.932

29. Staedt J, Stoppe G, Kögler A, et al. Dopamine D2 receptor alteration in patients with periodic movements in sleep (nocturnal myoclonus). J Neural Transm. 1993;93(1):71-74. doi:10.1007/BF01244940

30. Ruottinen HM, Partinen M, Hublin C, et al. An FDOPA PET study in patients with periodic limb movement disorder and restless legs syndrome. Neurology. 2000;54(2):502. doi:10.1212/WNL.54.2.502

31. Aggarwal S, Dodd S, Berk M. Restless leg syndrome associated with atypical antipsychotics: current status, pathophysiology, and clinical implications. Curr Drug Saf. 2015;10(2):98-105. doi:10.2174/ 1574886309666140527114159

32. Ruppert E, Bataillard M, Namer IJ, et al. Hyperdopaminergism in lenticulostriate stroke-related restless legs syndrome: an imaging study. Sleep Med. 2017;30:136-138. doi:10.1016/j.sleep.2016.02.011

33. Happe S, Pirker W, Klösch G, Sauter C, Zeitlhofer J. Periodic leg movements in patients with Parkinson's disease are associated with reduced striatal dopamine transporter binding. J Neurol. 2003;250 (1):83-86. doi:10.1007/s00415-003-0957-8

34. Puligheddu M, Figorilli M, Aricò D, Raggi A, Marrosu F, Ferri R. Time structure of leg movement activity during sleep in untreated Parkinson disease and effects of dopaminergic treatment. Sleep Med. 2014;15(7):816-824. doi:10.1016/j.sleep.2014.03.011

35. Bliwise DL, Trotti LM, Yesavage JA, Rye DB. Periodic leg movements in sleep in elderly patients with Parkinsonism and Alzheimer's disease. Eur J Neurol. 2012;19(6):918-923. doi:10.1111/j.1468-1331. 2012.03673.x

36. Lee W-T, Huang H-L, Wong LC, et al. Tourette syndrome as an independent risk factor for subsequent sleep disorders in children: a nationwide population-based case-control study. Sleep. 2017;40(3). doi:10.1093/sleep/zsw072

37. Friedman M, Wilson M, Lin H-C, Chang H-W. Updated systematic review of tonsillectomy and adenoidectomy for treatment of pediatric obstructive sleep apnea/hypopnea syndrome. Otolaryngol Neck Surg. 2009;140(6):800-808. doi:10.1016/j.otohns.2009.01.043

38. Cocuzza S, Marino S, Gulino A, et al. ENT involvement and orobuccal movements' disorders in Pandas patients: assessment and rehabilitations tools. Eur Rev Med Pharmacol Sci. 2019;23 (10):4110-4117. doi:10.26355/eurrev 201905 17912

39. Ryan MA, Leu GR, Boss EF, Raynor EM, Walsh JM. Adherence to American Academy of Otolaryngology-head and neck surgery clinical practice guidelines: a systematic review. Otolaryngol Head Neck Surg. 2020;163:194599820922155. doi:10.1177/0194599820922155

40. Pietropaoli N, Supino MC, Vitelli O, et al. Cognitive function in preschool children with sleep-disordered breathing. Sleep Breath 2015;19(4):1431-1437. doi:10.1007/s11325-015-1157-3

41. Kheirandish-Gozal L, Gozal D. Pediatric osa syndrome morbidity biomarkers: the hunt is finally on! Chest. 2017;151(2):500-506. doi:10.1016/j.chest.2016.09.026

42. Arnulf I, Groos E, Dodet P. Kleine-Levin syndrome: a neuropsychiatric disorder. Rev Neurol (Paris). 2018;174 (4):216-227. doi:10.1016/j.neurol.2018.03.005

43. Iranzo A. Sleep and neurological autoimmune diseases. Neuropsychopharmacology. 2020;45(1):129-140. doi:10.1038/s41386019-0463-z

44. Michiels V, Cluydts R. Neuropsychological functioning in chronic fatigue syndrome: a review. Acta Psychiatr Scand. 2001;103 (2):84-93. doi:10.1034/j.1600-0447.2001.00017.x

45. Nijhof LN, Nijhof SL, Bleijenberg G, et al. The impact of chronic fatigue syndrome on cognitive functioning in adolescents. Eur J Pediatr. 2016;175(2):245-252. doi:10.1007/s00431-015-2626-1

46. Yelland GW. Gluten-induced cognitive impairment ("brain fog") in coeliac disease. J Gastroenterol Hepatol. 2017;32:90-93. doi:10.11 11/jgh.13706
47. van Langenberg DR, Yelland GW, Robinson SR, Gibson PR. Cognitive impairment in Crohn's disease is associated with systemic inflammation, symptom burden and sleep disturbance. United Eur Gastroenterol J. 2017;5(4):579-587. doi:10.1177/205064061666 3397

48. Ibarra-Coronado EG, Pantaleón-Martínez AM, Velazquéz-Moctezuma $\mathrm{J}$, et al. The bidirectional relationship between sleep and immunity against infections. J Immunol Res. 2015;2015. doi:10.1155/2015/678164

49. Silber MH. Autoimmune Sleep Disorders. Vol. 133. 1st. Elsevier B. V.; 2016. doi:10.1016/B978-0-444-63432-0.00018-9

50. Haque A, Polcyn R, Matzelle D, Banik NL. New insights into the role of neuron-specific enolase in neuro-inflammation, neurodegeneration, and neuroprotection. Brain Sci. 2018;8(2):33. doi:10.3390/ brainsci8020033

51. Benedict C, Cedernaes J, Giedraitis V, et al. Acute sleep deprivation increases serum levels of neuron-specific enolase (NSE) and S100 calcium binding protein B (S-100B) in healthy young men. Sleep. 2014;37(1):195-198. doi:10.5665/sleep.3336

52. Zhang P, Tan C-W, Chen G-H, et al. Patients with chronic insomnia disorder have increased serum levels of neurofilaments, neuron-specific enolase and S100B: does organic brain damage exist? Sleep Med. 2018;48:163-171. doi:10.1016/j. sleep.2017.12.012

53. Kotagal S. Sleep-wake disorders of childhood. Contin Lifelong Learn Neurol. 2017;23(4):1132-1150. doi:10.1212/CON.0000000000000504

54. Howard H, Kamat D. restless legs syndrome in children. Pediatr Ann. 2018;47(12):e504-e506. doi:10.3928/19382359-20181114-02

55. Earley CJ, Connor J, Garcia-Borreguero D, et al. Altered Brain iron homeostasis and dopaminergic function in Restless Legs Syndrome (Willis-Ekbom Disease). Sleep Med. 2014;15(11):1288-1301. doi:10.1016/j.sleep.2014.05.009

56. Trotti LM. Restless legs syndrome and sleep-related movement disorders. Continuum (Minneap Minn). 2017;23(4,Sleep Neurology):1005-1016. doi:10.1212/CON.0000000000000488

57. Gao Q, Kou T, Zhuang B, Ren Y, Dong X, Wang Q. The association between vitamin D deficiency and sleep disorders: a systematic review and meta-analysis. Nutrients. 2018;10(10):1395. doi:10.33 90/nu10101395

58. Yang C-Y, Leung PSC, Adamopoulos IE, Gershwin ME. The implication of vitamin D and autoimmunity: a comprehensive review. Clin Rev Allergy Immunol. 2013;45(2):217-226. doi:10.1007/s12016-0138361-3

59. Peelen E, Knippenberg S, Muris A-H, et al. Effects of vitamin D on the peripheral adaptive immune system: a review. Autoimmun Rev. 2011;10(12):733-743. doi:10.1016/j.autrev.2011.05.002

60. Hewison M. An update on vitamin D and human immunity. Clin Endocrinol (Oxf). 2012;76(3):315-325. doi:10.1111/j.1365-2265.20 11.04261.x

61. Gunville CF, Mourani PM, Ginde AA. The role of vitamin D in prevention and treatment of infection. Inflamm Allergy Drug Targets. 2013;12(4):239-245. doi:10.2174/18715281113129990046

62. Qiu M-H, Vetrivelan R, Fuller PM, Lu J. Basal ganglia control of sleep-wake behavior and cortical activation. Eur J Neurosci. 2010;31 (3):499-507. doi:10.1111/j.1460-9568.2009.07062.x

63. Björklund A, Dunnett SB. Dopamine neuron systems in the brain: an update. Trends Neurosci. 2007;30(5):194-202. doi:10.1016/j.tins.20 07.03.006

64. Schultz W. Behavioral dopamine signals. Trends Neurosci. 2007;30 (5):203-210. doi:10.1016/j.tins.2007.03.007

65. Nambu A. Seven problems on the basal ganglia. Curr Opin Neurobiol. 2008;18(6):595-604. doi:10.1016/j.conb.2008.11.001

66. Oishi Y, Lazarus M. The control of sleep and wakefulness by mesolimbic dopamine systems. Neurosci Res. 2017;118:66-73. doi:10.1016/j.neures.2017.04.008 
67. Volkow ND, Wang G-J, Telang F, et al. Sleep deprivation decreases binding of $[11 \mathrm{C}]$ raclopride to dopamine D2/D3 receptors in the human brain. J Neurosci. 2008;28(34):8454-8461. doi:10.1523/ JNEUROSCI.1443-08.2008

68. Ma C, Zhong P, Liu D, et al. Sleep regulation by neurotensinergic neurons in a thalamo-amygdala circuit. Neuron. 2019;103(2):323334.e7. doi:10.1016/j.neuron.2019.05.015

69. Crittenden JR, Graybiel AM. Basal ganglia disorders associated with imbalances in the striatal striosome and matrix compartments. Front Neuroanat. 2011;5. doi:10.3389/fnana.2011.00059.

70. Alexander GE, Crutcher MD, DeLong MR. Basal gangliathalamocortical circuits: parallel substrates for motor, oculomotor, "prefrontal" and "limbic" functions. Prog Brain Res. 1990;85:119-146.

71. Haber SN. The primate basal ganglia: parallel and integrative networks. J Chem Neuroanat. 2003;26(4):317-330. doi:10.1016/j. jchemneu.2003.10.003
72. Siegel M, Beresford CA, Bunker M, et al. Preliminary investigation of lithium for mood disorder symptoms in children and adolescents with autism spectrum disorder. J Child Adolesc Psychopharmacol. 2014;24(7):399-402. doi:10.1089/cap.2014.0019

73. Blanchard JJ, Andrea A, Orth RD, Savage C, Bennett ME. Sleep disturbance and sleep-related impairment in psychotic disorders are related to both positive and negative symptoms. Psychiatry Res. 2020;286:112857. doi:10.1016/j.psychres.2020.112857

74. Calaprice D, Tona J, Parker-Athill EC, Murphy TK. A survey of pediatric acute-onset neuropsychiatric syndrome characteristics and course. J Child Adolesc Psychopharmacol. 2017;27(7):607-618. doi:10.1089/cap.2016.0105

\section{Publish your work in this journal}

Nature and Science of Sleep is an international, peer-reviewed, open access journal covering all aspects of sleep science and sleep medicine, including the neurophysiology and functions of sleep, the genetics of sleep, sleep and society, biological rhythms, dreaming, sleep disorders and therapy, and strategies to optimize healthy sleep.
The manuscript management system is completely online and includes a very quick and fair peer-review system, which is all easy to use. Visit http://www.dovepress.com/testimonials.php to read real quotes from published authors. 